\title{
Nanog Is an Essential Factor for Induction of Pluripotency in Somatic Cells from Endangered Felids
}

\author{
Rajneesh Verma, Jun Liu, Michael Kenneth Holland,2 Peter Temple-Smith,,3 \\ Mark Williamson, ${ }^{4}$ and Paul John Verma ${ }^{1,5}$
}

\begin{abstract}
Nanog has an important role in pluripotency induction in bovines and snow leopards. To examine whether it was required for wild felids globally, we examined the induction of pluripotency in felids from Asia (Bengal tiger, Panthera tigris), Africa (serval, Leptailurus serval), and the Americas (jaguar, Panthera onca). Dermal fibroblasts were transduced with genes encoding the human transcription factors OCT4, SOX2, KLF4, and cMYC with or without NANOG. Both four- and five-factor induction resulted in colony formation at day 3 in all three species tested; however, we were not able to maintain colonies that were generated without NANOG beyond passage (P) 7. Five-factor induced pluripotent stem cell (iPSC) colonies from wild cats were expanded in vitro on feeder layers and were positive for alkaline phosphatase and protein expression of OCT-4, NANOG, and stage-specific embryonic antigen-4 at P4 and P14. Reverse-transcription polymerase chain reaction confirmed that all five human transgenes were transcribed at P4; however, OCT4, SOX2, and NANOG transgenes were silenced by P14. Endogenous OCT4 and NANOG transcripts were detected at P4 and P14 in all cell lines confirming successful reprogramming. At P14, the iPSCs from all three species remained euploid and differentiated in vivo and in vitro into derivatives of the three germ layers. This study describes an effective method for inducing pluripotency in three endangered wild cats from across the globe and confirms Nanog as an essential factor in the reprogramming event. Efficient production of iPSC from endangered felids creates a unique opportunity for species preservation through gamete production, nuclear transfer, embryo complementation, and future novel technologies.
\end{abstract}

Key words: conservation; induced pluripotent stem cells; Nanog; wild cats

\section{Introduction}

I NDUCED PLURIPOTENCY IS A NEW APPROACH to produce embryonic stem-like cells from somatic cells ${ }^{1}$ and provides a unique approach to understand both pluripotency and lineage assignment. Generation of induced pluripotent stem cells (iPSCs) from endangered species ${ }^{2,3}$ raises fascinating possibilities for species conservation by augmenting the available technologies, such as nuclear transfer and embryo complementation, for assisted reproduction. However, some challenges hinder this application, with the primary challenge being limited availability of compatible recipient eggs and surrogate dams for embryos generated, ${ }^{4}$ though recent advances in the field hold promise. ${ }^{5,6}$ In the Felidae, endangered and nonendangered species can interbreed to produce viable hybrids (e.g., tigon, jaglion, Savannah cat), demonstrating embryo-uterine and mitochondrial compatibility for embryo surrogacy. ${ }^{7}$ This circumvents the potential barriers in selected endangered felids for using iPSCs to assist reproduction.

iPSCs with properties that are equivalent to embryonic stem cells (ESCs) have been generated in rodents, primates, and some domestic species. ${ }^{8-11}$ In most domestic species, however, maintenance of iPSCs is dependent on continued expression of exogenous transgenes ${ }^{12}$ reflecting species differences in pluripotency, a factor potentially also restricting isolation of ESCs from these species.

We have recently identified the critical role of Nanog in pluripotency induction and maintenance in some large animal species. ${ }^{3,11}$ To determine whether Nanog was an absolute

\footnotetext{
${ }^{1}$ Center for Reproduction and Development, Monash Institute of Medical Research; ${ }^{3}$ Department of Obstetrics and Gynecology, Southern Clinical School; Monash University, Clayton, Australia.

${ }^{2}$ School of Veterinary Science, The University of Queensland, Gatton, Australia.

${ }^{4}$ Gribbles Pathology, Clayton, Australia.

${ }^{5}$ South Australian Research and Development Institute, Turretfield Research Center, Rosedale, Australia.
} 
requirement for robust derivation of iPSCs from felids globally, we examined the induction of pluripotency in fibroblast cultures from three geographically separated and evolutionarily diverse felid species: $:^{13}$ the Asian Bengal tiger (Panthera tigris), the African serval (Leptailurus serval), and the South American jaguar (Panthera onca).

\section{Materials and Methods}

Animal handling and experimentation was conducted in accordance with the code of practice of the Australian National Health and Medical Research Council (NHMRC; 2004) and was approved by Monash University Animal Experimentation Ethics Committee.

Fibroblasts were isolated from tissues taken from the ear pinnae of Bengal tiger, jaguar, and serval, which died of natural causes or were euthanized due to health-related problems. The tissue $\left(\sim 5 \mathrm{~mm}^{2}\right)$ was minced using sterile surgical instruments and plated in six-well dishes with fibroblast plating (FP) medium. The FP medium was composed of Dulbecco's modified Eagle's medium (DMEM) with high glucose supplemented with penicillin/streptomycin and $10 \%(\mathrm{v} / \mathrm{v})$ fetal bovine serum (all from Invitrogen, Mulgrave, Australia) The tissue was cultured in a $6 \% \mathrm{CO}_{2}$ incubator at $38.5^{\circ} \mathrm{C}$ for 7 days. ${ }^{10}$

Mouse embryonic fibroblasts (MEFs) were isolated from fetuses collected from Quackenbush (QS) mice on day 13.5 post-coitum and used as a feeder layer for the iPSCs as previously described. ${ }^{3}$ QS-MEFs were cultured in MEF medium which consisted of DMEM (high glucose) supplemented with $10 \%(\mathrm{v} / \mathrm{v})$ fetal bovine serum, $2 \mathrm{mM}$ Glutamax, penicillin/ streptomycin, and $1 \mathrm{M}$ nonessential amino acids (Invitrogen). MEFs were treated with $10 \mu \mathrm{g} / \mathrm{mL}$ mitomycin C (Invitrogen) for $3 \mathrm{~h}$ to arrest mitosis. ${ }^{14}$

Moloney-based retroviral vectors (pMXs) containing the coding sequences of GFP, human OCT4, SOX2, KLF4, $C M Y C$, and NANOG genes were obtained from Addgene (Cambridge, MA). Amphotropic viral particle production by Plat-A packaging cells (Jomar Biosciences, Kensington, Australia) was performed as described previously. ${ }^{3}$ Nine micrograms of each vector was co-transfected to Plat-A cells using Fugene 6 (Roche, Dee Why, Australia) according to manufacturer's instruction. Virus-containing supernatants were collected 48 and $72 \mathrm{~h}$ post-transfection and filtered through a $0.45-\mu \mathrm{m}$ pore-size filter and supplemented with $8 \mu \mathrm{g} / \mathrm{mL}$ of polybrene (Sigma-Aldrich, St. Louis, MO). Felids fibroblasts were plated $24 \mathrm{~h}$ prior to infection at a density of $4 \times 10^{4}$ cells $/ \mathrm{cm}^{2}$. Equal parts of the retroviral supernatant containing four or five transcription factors were added to the plated felid fibroblasts. Two rounds of infection were performed $24 \mathrm{~h}$ apart. The culture medium was changed to mouse ESC medium at day 5 post-infection. The iPSC colonies were picked based on ESC-like morphology at day 3. Felids fibroblast cells were infected with GFP-containing retroviral supernatant in parallel, to monitor the transduction efficiency.

The felid iPSCs were cultured in medium consisting of DMEM supplemented with $0.1 \mathrm{mmol} / \mathrm{L}$ 2-beta-mercaptoethanol, $1 \mathrm{M}$ nonessential amino acid, $2 \mathrm{mM}$ Glutamax, $20 \%$ (v/v) Hyclone serum, penicillin/streptomycin, and $10^{3} \mathrm{U} /$ $\mathrm{mL}$ murine leukemia inhibitory factor (LIF) (ESGRO; Invitrogen) in a $6 \% \mathrm{CO}_{2}$ incubator at $38.5^{\circ} \mathrm{C}$. Medium was changed daily, and iPSCs were passaged manually every $3-4$ days onto fresh feeder layers.
Putative iPSC colonies were tested for alkaline phosphatase (AP) using a diagnostic AP substrate kit according to the manufacturer's specification (SK-5300, Vector Laboratories, Inc., Burlingame, CA). For immunocytochemistry, colonies were fixed in $40 \mathrm{mg} / \mathrm{mL}$ paraformaldehyde for $15 \mathrm{~min}$ at room temperature and washed three times with phosphate-buffered saline (PBS, pH 7.5). Cells examined for OCT-4 and NANOG were blocked with PBS (pH 7.5) supplemented with $10 \mathrm{mg} / \mathrm{mL}$ bovine serum albumin (BSA), $5 \%$ $(\mathrm{v} / \mathrm{v})$ goat serum, and $0.1 \%(\mathrm{v} / \mathrm{v})$ Tween. Those tested for stage-specific embryonic antigen (SSEA)-4 were blocked with $10 \mathrm{mg} / \mathrm{mL}$ BSA, $5 \%(\mathrm{v} / \mathrm{v})$ goat serum in PBS pH 7.5 for $60 \mathrm{~min}$ at room temperature. Mouse anti-human OCT-4 immunoglobulin G (IgG; SC-5279, Santa Cruz Biotechnology, Santa Cruz, CA), mouse anti-mouse SSEA-4 IgG (MC 813-70, Millipore, Melbourne, Australia) and rabbit polyclonal IgG to NANOG IgG (ab-80892, Abcam, Waterloo, Australia) were used as the primary antibodies at 1:100 dilution in PBS containing $5 \%(\mathrm{v} / \mathrm{v})$ goat serum in an overnight incubation at $4^{\circ} \mathrm{C}$. Cell preparations were then washed three times with PBS and a secondary antibody was added in PBS containing $5 \%(\mathrm{v} / \mathrm{v})$ goat serum for $1 \mathrm{~h}$ at room temperature (OCT-4: anti-IgG Alexa 488 1:500; NANOG: anti-IgG Alexa 488 1:500; SSEA-4: anti-IgG Alexa 594 1:500). In addition, control cell lines with the primary antibodies excluded were used as negative controls. At the end of the secondary antibody reaction all cell preparations were washed three times with PBS, mounted in Vectashield +4',6-diamidino-2-phenylindole (DAPI; Abacus) and cover-slipped.

Total RNA was extracted from felid iPSCs at passage (P) 4 and P14, and also from human ESC, mouse ESC, and tiger, jaguar, and serval ear fibroblasts using an RNA-easy kit (Qiagen, Doncastor, VIC, Australia) according to the manufacturer's instructions. We used GAPDH expression as an internal control for the amount of template in each reaction. Polymerase chain reaction (PCR) was carried out using Go Taq DNA polymerase (Sigma). Primers used in this study ${ }^{15}$ are listed in Supplementary Tables S1 and S2.

To test for embryoid bodies (EBs) formation, felid iPSCs at P14 were typsinized and $20 \times 10^{3}$ cells were plated in lowattachment plastic six-well dishes. iPSC medium was used without LIF and was changed every second day till day $10 .{ }^{16}$ EBs were collected after 10 days for RNA extraction and preparation of cDNAs for reverse-transcription (RT)-PCR. The primers used for detecting three germ layers were Nestin (endoderm), CD 31 (mesoderm), and FoxA2 (ectoderm). ${ }^{17}$

Felid iPSCs at P14 were harvested using $5 \mathrm{mg} / \mathrm{mL}$ trypsinEDTA (Invitrogen). Two million cells were injected subcutaneously into the thigh muscle of 5- to 7-week-old severe combined immunodeficient (SCID) male mice. ${ }^{17}$ Ten weeks after injection the mice were euthanized and the resulting tumors were harvested and fixed in $40 \mathrm{mg} / \mathrm{mL}$ paraformaldehyde, embedded in paraffin, sectioned at $5 \mu \mathrm{m}$, and stained with hematoxylin and eosin.

At least 60 metaphase spreads were counted for tiger, jaguar, and serval iPSCs (TiPSCs, JiPSCs, and SiPSCs, respectively) at P14 for karyotype analysis. ${ }^{3}$

\section{Results and Discussion}

Moloney-based retroviral vectors (pMXs) encoding the human transcription factors OCT4, SOX2, KLF4, and $c M Y C$ 
A
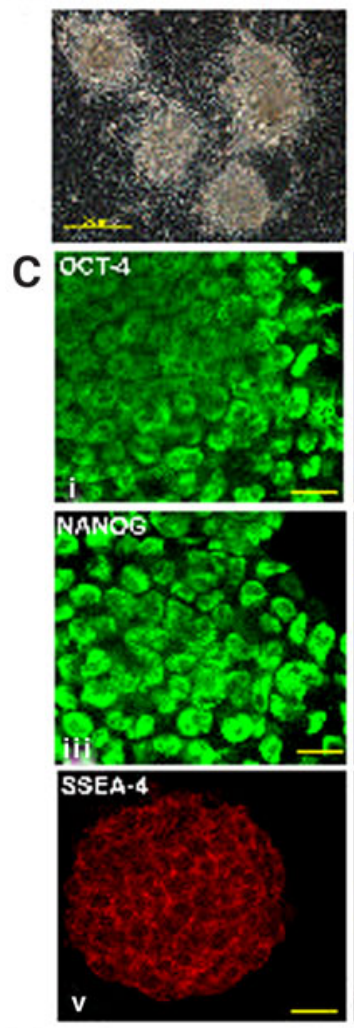

F

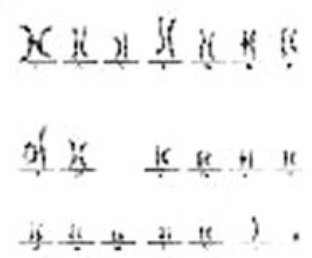

H

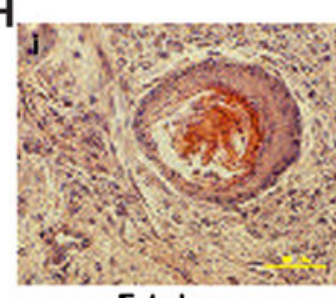

Ectoderm
B
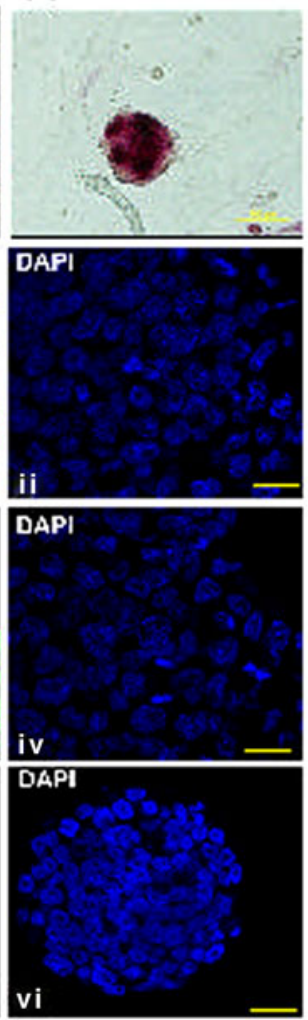

G
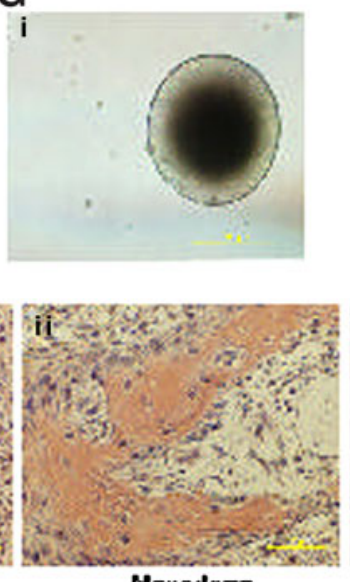

D

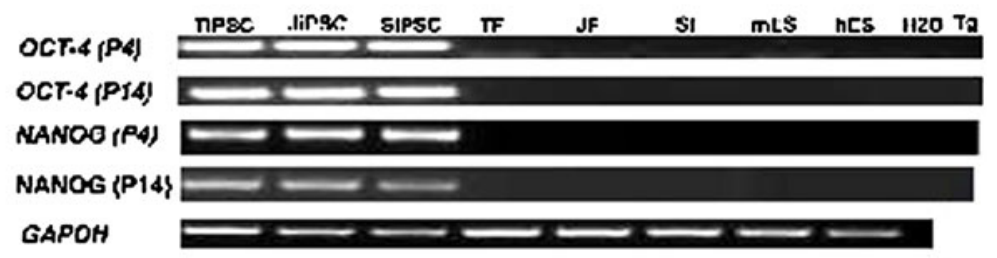

Passage 4

$\mathbf{E}$

OCT-4 TIPEC MIPSE SII'SE TH TF JF SF MES hES H2O

NANOC

sax-2

Ni $F-1$

arsc

\section{ii Passage 14}

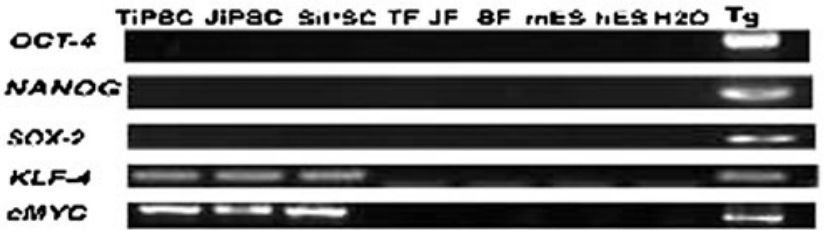

iii

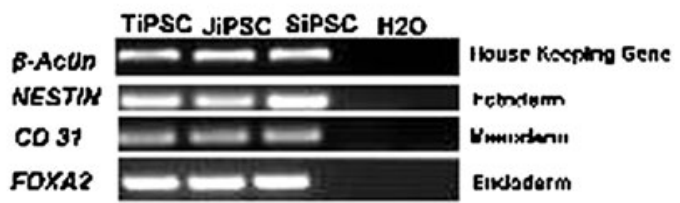

FIG. 1. Characteristics of Bengal tiger induced pluripotent stem cells (TiPSCs). (A) Morphology of TiPSCs at P1. (B) Alkaline phosphatase (AP) staining of a putative TiPSC colony at P14. (C) Confocal images of immunofluorescence staining of TiPSC at P14. OCT-4: (i) green fluorescence, (ii) 4',6-diamidino-2-phenylindole (DAPI); NANOG: (iii) green fluorescence, (iv) DAPI; stage-specific embryonic antigen (SSEA)-4: (v) red fluorescence, (vi) DAPI. The scale bar for all confocal images represents $25 \mu \mathrm{m}$. (D) Reverse-transcription polymerase chain reaction (RT-PCR) gene expression analysis of endogenous cat OCT4 (286 bp), NANOG (246 bp), and GAPDH (271 bp) at P4 and P14 in Bengal tiger, jaguar, and serval iPSCs (TiPSCs, JiPSCs, and SiPSCs, respectively) and fibroblasts (TFs, JFs, and SFs), mouse and human embryonic stem cells (mESCs and hESCs), transgene $(\mathrm{Tg})$, and water $\left(\mathrm{H}_{2} \mathrm{O}\right)$. (E, F) Expression of human transgenes pMX-OCT-4, pMX-NANOG, pMX-SOX-2, pMXKLF-4, and pMX-cMYC in TiPSCs, JiPSCs, SiPSCs, Tg, TFs, JFs, SFs, mESCs, hESCs, and $\mathrm{H}_{2} \mathrm{O}$ at P4 and P14. (G) Karyotype of TiPSCs at P14 showing a euploid count of 18 autosomal and one XY pair of chromosomes. (H) TiPSCs differentiated in vitro to form embryoid bodies. (I) Confirmation of differentiation of embryoid bodies into the three germ layers by specific gene expression by RT-PCR (housekeeping gene: $\beta$-actin; ectoderm: NESTIN; mesoderm: CD31; and endoderm: FOXA2) on TiPSCs, JiPSCs, SiPSCs, and $\mathrm{H}_{2} \mathrm{O}$. (J) Cross-section of a teratoma obtained at 10 weeks after injection of TiPSCs at P14 in a severe combined immunodeficient (SCID) mouse, stained with hematoxylin and eosin with tissues representative of the three primary germ layers: (i) ectoderm: keratinizing stratified squamous epithelium; (ii) mesoderm: bone surrounded by connective tissue; (iii) endoderm: ciliated epithelium. 
with or without NANOG were used to infect dermal fibroblasts from each species. Infection efficiency of the retrovirus was ascertained using pMX-GFP transgene expression and averaged $91.3 \% \pm 1.2 \%, 92.3 \% \pm 1.2 \%$, and $98.3 \% \pm 0.6 \%$ in tiger, jaguar, and serval, respectively, from three repeated experiments (Supplementary Table S3). Both four- and fivefactor induction initiated colony formation at day 3 ; however, colony formation and programming efficiency were reduced in four-factor inductions (SupplementaryTable S3) and we were not able to maintain colonies that were generated without NANOG beyond seven passages (P7).

Five-factor-induced iPSC colonies from tiger, jaguar, and serval were expanded in vitro (Figs. $1 \mathrm{~A}$ and $2 \mathrm{~A}, \mathrm{~A}^{\prime}$ ) and were positive for $\mathrm{AP}$ (Figs. $1 \mathrm{~B}$ and $2 \mathrm{~B}, \mathrm{~B}^{\prime}$ ) and protein expression of OCT-4, NANOG, and SSEA-4 at P14 (Figs. 1C and $\left.2 \mathrm{C}, \mathrm{C}^{\prime}\right)$. Endogenous OCT-4 and NANOG were detected by RT-PCR at P4 and P14 (Fig. 1D) indicating reprogramming and reactivation of the endogenous pluripotency genes. RT-PCR confirmed that all five transgenes were transcribed at P4 (Fig. 1E); however, OCT-4, SOX-2, and NANOG transgenes were silenced by P14 (Fig. 1F). Karyotypes of the iPSC lines (Figs. 1G and 2D, D') were normal (18XX or 18XY) in all three species. Under in vitro differentiation conditions, embryoid bodies developed from all iPSC lines (Figs. $1 \mathrm{H}$ and $\left.2 \mathrm{E}, \mathrm{E}^{\prime}\right)$ and randomly differentiated into ectoderm, endoderm, and mesoderm as detected by RT-PCR of specific marker genes (Fig. 1I). Further, when injected into SCID mice, P14 iPSCs from all three felid species formed teratomas containing tissues from the three germ layers (Figs. 1J and 2F, $\left.\mathrm{F}^{\prime}\right)$.

In conclusion, we describe an effective method for inducing pluripotency in cells from large endangered felids and confirm Nanog as an essential factor in the reprogramming milieu for these species. Felids are also suitable models for studying diseases, including HIV, but lack of germ-linecompetent ESCs has limited the ability for genome modification in the cat. ${ }^{7}$ The efficient production of iPSCs from felids therefore creates a novel opportunity for species preservation through assisted reproduction and provides an alternative for elusive cat ESCs for biomedical research.
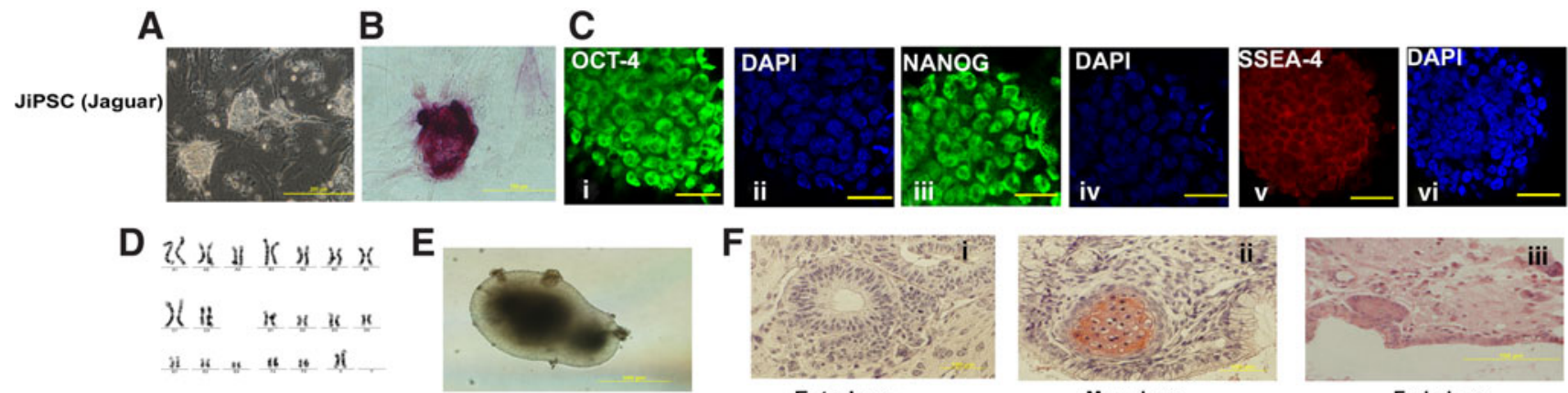

Ectoderm
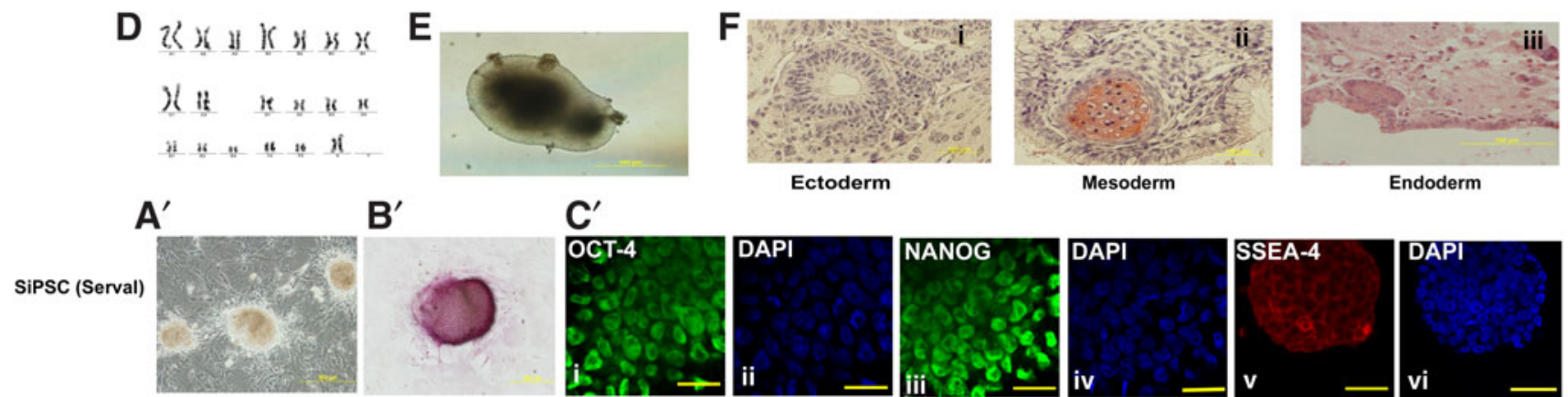

$B^{\prime}$
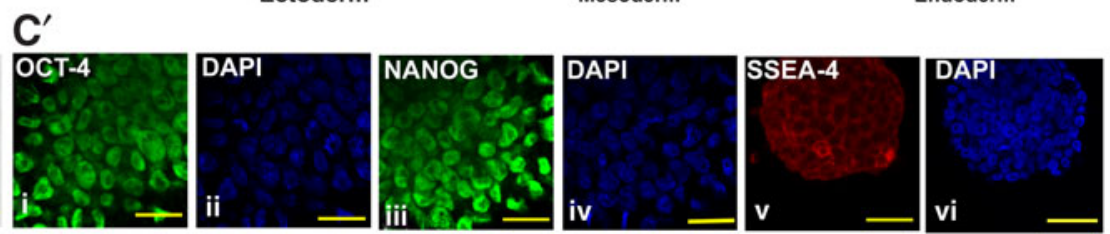

$\mathbf{D}^{\prime}$

$\mathbf{E}^{\prime}$
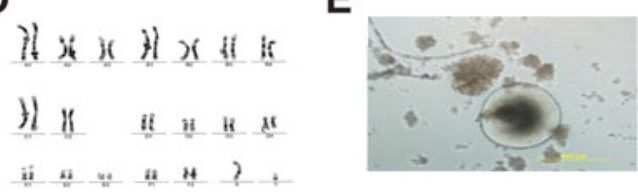

$F^{\prime}$
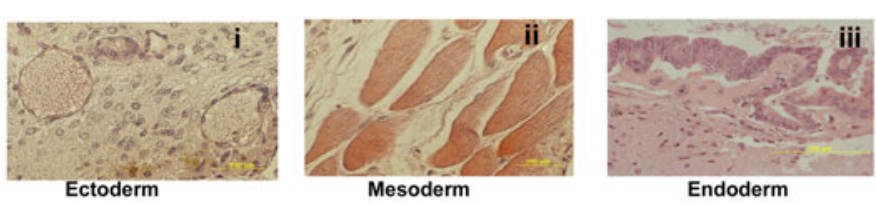

FIG. 2. Detailed characterization of JiPSCs and SiPSCs at P14. (A-F) Morphology and characterization of JiPSCs. (A) Morphology of JiPSCs at P1 on feeder layers. (B) AP staining of a putative JiPSC colony at P14. (C) Confocal images of immunofluorescence staining of JiPSCs at P14. OCT-4: (i) green fluorescence, (ii) DAPI; NANOG: (iii) green fluorescence, (iv) DAPI; SSEA-4: (v) red fluorescence, (vi) DAPI. The scale bar for all confocal images represents $25 \mu \mathrm{m}$. (D) Karyotype of JiPSCs at P14 showing a euploid count of 18 autosomal and one XX pair of chromosomes. (E) JiPSC differentiated in vitro to form embryoid bodies. (F) Cross-section of a teratoma obtained at 9 weeks after injection of JiPSCs at P14 in a SCID mouse, stained with hematoxylin and eosin with tissues representative of the three primary germ layers: (i) ectoderm: nervous tissue; (ii) mesoderm: bone surrounded by connective tissue; (iii) endoderm: cuboidal epithelium. ( $\left.\mathbf{A}^{\prime}-\mathbf{F}^{\prime}\right)$ Morphology and characterization of SiPSCs. (A') Morphology of SiPSC at P1 on feeder layers. (B') AP staining of a putative SiPSC colony at P14. (C') Confocal images of immunofluorescence staining of SiPSCs at P14: OCT-4: (i) green fluorescence, (ii) DAPI; NANOG: (iii) green fluorescence, (iv) DAPI; SSEA-4: (v) red fluorescence, (vi) DAPI. The scale bar for all confocal images represents $25 \mu \mathrm{m}$. (D') Karyotype of SiPSCs at P14 showing a euploid count of 18 autosomal and one XY pair of chromosomes; (E') SiPSCs differentiated in vitro to form embryoid bodies. $\left(F^{\prime}\right)$ Cross-section of a teratoma obtained at 9 weeks after injection of SiPSCs at P14 in a SCID mouse, stained with hematoxylin and eosin with tissues representative of the three primary germ layers: (i) ectoderm: nervous tissue with blood cells (vascular differentiation); (ii) mesoderm: muscle; (iii) endoderm: columnar epithelium. 


\section{Acknowledgments}

We thank Ms. Sally Padey (Director) and Ms. Hannalie Vander Merwe (Officer-in-charge) of Mogo Zoo in New South Wales (Mogo, Australia) for their generous support to this project by providing tissue samples. We also thank all the staff at Mogo Zoo and the zoo veterinarians, Dr. Mary Atkinson and Dr. Peter Atkinson. Rajneesh Verma acknowledges a PhD scholarship awarded by Professor Bryan Williams and the Monash Institute of Medical Research (Melbourne, Australia). This research was also supported by the Victorian Government's Operational Infrastructure Support Program.

\section{Author Disclosure Statement}

All authors declare that no competing financial interests exist.

\section{References}

1. Takahashi K, Yamanaka S. Induction of pluripotent stem cells from mouse embryonic and adult fibroblast cultures by defined factors. Cell. 2006;663-676.

2. Ben-Nun IF, Montague SC, Houck ML, et al. Induced pluripotent stem cells from highly endangered species. Nat Methods. 2011;8:829-831.

3. Verma R, Holland MK, Temple-Smith $\mathrm{P}$, et al. Inducing pluripotency in somatic cells from the snow leopard (Panthera uncia), an endangered felid. Theriogenology. 2012; 77:220-228, 228 e221-222.

4. Selvaraj V, Wildt DE, Pukazhenthi BS. Induced pluripotent stem cells for conserving endangered species? Nat Methods. 2011;8:805-807.

5. Hayashi K, Ogushi S, Kurimoto K, et al. Offspring from oocytes derived from in vitro primordial germ cell-like cells in mice. Science. 2012;338:971-975.

6. Hayashi K, Ohta H, Kurimoto K, et al. Reconstitution of the mouse germ cell specification pathway in culture by pluripotent stem cells. Cell. 2011;146:519-532.

7. Wongsrikeao P, Saenz D, Rinkoski T, et al. Antiviral restriction factor transgenesis in the domestic cat. Nat Methods. 2011;8:853-859.
8. Esteban MA, Wang T, Qin B, et al. Vitamin C enhances the generation of mouse and human induced pluripotent stem cells. Cell Stem Cell. 2010;6:71-79.

9. Liu J, Sumer H, Leung J, et al. Late passage human fibroblasts induced to pluripotency are capable of directed neuronal differentiation. Cell Transplant. 2011;20:193-203.

10. Tat PA, Sumer H, Jones KL, et al. The efficient generation of induced pluripotent stem (iPS) cells from adult mouse adipose tissue-derived and neural stem cells. Cell Transplant. 2010;19:525-536.

11. Sumer H, Liu J, Malaver-Ortega LF, et al. NANOG is a key factor for induction of pluripotency in bovine adult fibroblasts. J Anim Sci. 2011;89:2708-2716.

12. Ezashi T, Telugu BP, Alexenko AP, et al. Derivation of induced pluripotent stem cells from pig somatic cells. Proc Natl Acad Sci USA. 2009;106:10993-10998.

13. Werdelin L, Lewis ME. Plio-Pleistocene carnivora of eastern Africa: species richness and turnover patterns. Zool J Linn Soc-Lond. 2005;144:121-144.

14. Sumer H, Jones KL, Liu J, et al. Reprogramming of somatic cells after fusion with induced pluripotent stem cells and nuclear transfer embryonic stem cells. Stem Cells Dev. 2010; 19:239-246.

15. Yu X, Jin G, Yin X, et al. Isolation and characterization of embryonic stem-like cells derived from in vivo-produced cat blastocysts. Mol Reprod Dev. 2008;75:1426-1432.

16. Liu J, Ashton MP, Sumer H, et al. Generation of stable pluripotent stem cells from NOD mouse tail-tip fibroblasts. Diabetes. 2011;60:1393-1398.

17. Liu J, Balehosur D, Murray B, et al. Generation and characterization of reprogrammed sheep induced pluripotent stem cells. Theriogenology. 2012;77:338-346 e331.

Address correspondence to: Paul John Verma, BSc, MSc, PhD Monash Inistitute of Medical Research Department of Reproduction and Development 27-31 Wright Street Clayton, VIC 3168 Australia

E-mail: paul.verma@monash.edu 